Rev. SINAPSIS, Edición № 12, Vol. 1, Diciembre 2018

ISSN 1390 - 9770

\title{
Gestión de las Tecnología de la Información y Comunicación en entidades educativas
}

Management of Information and Communication Technology in educational entities

Patricio Macay Dueñas, Ing. ${ }^{(1)}$

Daniela Vera Vélez, MSc. IE. ${ }^{(2)}$

Aurora Barreiro Vera, MSc. GE. ${ }^{(3)}$

Cristian Mera Macías, MSc. IE. ${ }^{(4)}$

(1) - (4) Universidad Laica Eloy Alfaro de Manabí Extensión Chone, Chone, Ecuador.

Contacto: angel.mera@uleam.edu.ec

\section{Receptado: 21/02/2018 Aceptado: 16/04/2018}

\section{Resumen}

El objetivo de esta investigación fue determinar el estado actual de la gestión de las TIC en una institución de educación secundaria del Cantón Tosagua, ubicada en la provincia de Manabí, República del Ecuador. Como problema se identificó el desconocimiento de la gestión interna de las Tecnologías de la Información y comunicación, Esta investigación es de tipo cualitativo de cohorte transversal. Los métodos utilizados son los teóricos, como el histórico lógico, deductivo inductivo, analítico sintético y el descriptivo además se utilizaron los métodos empíricos como la encuesta misma que se aplicó a 25 usuarios donde se pudo conocer: a) el nivel de implementación de procedimientos internos para el uso de las TIC, b) el nivel de conocimientos que tienen los usuarios para operar las TIC, c) la frecuencia con la que se capacita al personal en el uso de las TIC, d) los niveles de resolución de problemas y otros aspectos de la gestión de TIC, tales como las normas y/o estándares para dicha gestión.

Los recursos tecnológicos también conocidos como Tecnologías de la Información y Comunicación (TIC) incluyen el hardware, software, infraestructura de telecomunicaciones y bases de datos, estos elementos deben ser gestionados de forma correcta para que las organizaciones 
Rev. SINAPSIS, Edición № 12, Vol. 1, Diciembre 2018

puedan funcionar de manera eficiente, logrando con ello que tanto el usuario interno como el externo se empoderen en el uso de estas herramientas logrando un servicio de calidad.

Palabras clave: Gestión de TIC, Estándares de TIC, mejores prácticas de TIC

\begin{abstract}
The objective of this research was to determine the current state of ICT management in a secondary education institution of Cantón Tosagua, located in the province of Manabí, Republic of Ecuador. As a problem, the lack of knowledge of the internal management of Information and Communication Technologies was identified. This research is of a qualitative type of transversal cohort. The methods used are the theoretical ones, such as the logical historical, inductive deductive, synthetic analytical and descriptive. Empirical methods were also used, such as the survey itself applied to 25 users where it was possible to know: a) the level of implementation of internal procedures for the use of ICT, b) the level of knowledge that users have to operate ICT, c) the frequency with which staff is trained in the use of ICT, d) the levels of problem solving and other aspects of ICT management, such as standards and / or standards for such management. The technological resources also known as Information and Communication Technologies (ICT) include hardware, software, telecommunications infrastructure and databases, these elements must be managed correctly so that organizations can function efficiently, thereby achieving that both the internal and external users are empowered in the use of these tools, achieving a quality service.
\end{abstract}

Keyword: ICT management, ICT standards, ICT best practices

\title{
Introducción
}

La gestión tecnológica es un campo muy amplio, y actualmente es un aspecto fundamental para que las entidades ya sean públicas o privadas alcancen altos estándares de efectividad en sus actividades, la gestión tecnológica abarca la planeación, dirección, control y coordinación del desarrollo e implementación de capacidades tecnológicas para formular y alcanzar los objetivos estratégicos y operacionales de la organización, esta es una de las definiciones que recoge (Odremán, 2014). 
Rev. SINAPSIS, Edición № 12, Vol. 1, Diciembre 2018

En un trabajo presentado por (Aronica \& Peretti, 2010) se define a la gestión tecnológica como la disciplina en la que se mezclan conocimientos de ingeniería, ciencias y administración con el fin de realizar la planeación, el desarrollo y la implantación de soluciones tecnológicas que contribuyan al logro de los objetivos estratégicos y técnicos de una organización. Por otra parte (Navarro, Romero, Bauza, \& Granadillo, 2006) definen a la gestión de tecnología como el proceso mediante el cual una organización se percata de la existencia de una tecnología, la adquiere, la adapta a sus necesidades, obtiene avances en la misma y la abandona para procurar otra que satisfaga mejor sus necesidades y así incrementar o mantener su productividad. Este proceso es descrito como un ciclo continuo que puede ser aplicado a cualquier nivel dado: producto, servicio, función centro de trabajo, planta/supervisión, corporación e industria nacional o internacional. En términos generales, los procesos de gestión tecnológica en la empresa involucran funciones básicas, como: "identificación, evaluación y selección de tecnologías, desagregación de paquetes tecnológicos, negociación de tecnologías, construcción y puesta en marcha de sistemas productivos, uso y asimilación de tecnologías, adaptación y mejoramiento de la tecnología, generación y comercialización de nuevas tecnologías (Ávalos, 2003).

Según (Agudelo, Lina, Niebles, \& Gallón, 2005) la gestión tecnológica se constituye en una alternativa tanto estratégica como operativa que garantiza a las unidades de información su permanencia en el tiempo y en el espacio, asegurando su adaptación a los cambios globales e incrementando su capacidad competitiva, al desarrollar competencias que les permitan implementar de manera adecuada procesos de evaluación, transferencia y negociación de tecnologías y así mismo, un sistemas de indicadores de gestión que les faciliten la toma de decisiones en cuanto al desempeño de procesos y tecnologías en el interior de la organización, y a su vez, desarrollar estrategias para la identificación de las capacidades tecnológicas exógenas relacionadas con las oportunidades y amenazas tecnológicas del entorno. (Thamhain, 2005) explica que existen siete dimensiones involucradas en la gestión tecnológica:

Para (Cabrera, 2010), La gestión de tecnología es una de las disciplinas que en las pasadas tres décadas más se ha ocupado de estudiar y desarrollar la innovación empresarial, y la innovación tecnológica en particular. Se ha ocupado de la comprensión del fenómeno innovador, de su modelaje, de sus prácticas, de sus impactos en la competitividad empresarial, de sus actores, de sus procesos y prácticas, de la interacción de estos últimos con otros procesos y prácticas 
Rev. SINAPSIS, Edición № 12, Vol. 1, Diciembre 2018

ISSN 1390 - 9770

organizacionales, de su medición y evaluación, de sus técnicas y herramientas, y de las condiciones externas e internas necesarias para que sea efectiva en un contexto y un momento histórico particular.

- La gestión de la ingeniería, las ciencias naturales y las ciencias sociales.

- Las ciencias administrativas para la planeación, la selección, el desarrollo y la implementación de la tecnología.

- El desarrollo de capacidades operacionales y servicios de campo.

- Los procesos operacionales, herramientas, técnicas y personal.

- La dirección y el liderazgo hacia el desarrollo de nuevos productos y servicios.

- El ambiente de negocios, la cultura organizacional y la estrategia de negocios y su influencia recíproca.

- La gestión de muchos componentes interdisciplinarios, la gestión de su integración en un solo sistema y la gestión de dicho sistema

Para (Agudelo, Lina, Niebles, \& Gallón, 2005) la importancia estratégica de la información como resultado de la aparición de nuevos paradigmas tecnológicos, impone a las unidades de información la necesidad de buscar soluciones y responder a retos y exigencias sin precedentes, por lo tanto, se requiere que adopten un enfoque empresarial que les permita manejar adecuadamente su relación con el mercado, lo que significa su articulación en un entorno más competitivo de productos y servicios de información de mayor valor agregado.

Las entidades educativas no están exentas de las "buenas prácticas" que se deben aplicar en la gestión de las TIC. Para (Acevedo, 2010) las mejores prácticas son un conjunto de prácticas que alguien obtiene analizando y estudiando qué hacen y qué no hacen los mejores exponentes de un tema en particular. La idea es que al terminar el análisis se tendrá un conjunto de prácticas comunes a todos aquellos que están a la vanguardia, y es precisamente ese conjunto el que se recopila y se lanza como "las mejores prácticas" para un tema dado.

Según (María \& Pere, 2013), desde la investigación-acción pretenden impulsar la integración de tecnologías de la información y comunicación promoviendo la formación didáctica y tecnológica del profesorado para orientar el proceso hacia la experimentación del uso de las Tecnologías en las 
Rev. SINAPSIS, Edición № 12, Vol. 1, Diciembre 2018

ISSN 1390 - 9770

aulas. Por otro lado Sandoval, Arenas, López, Cabero y Aguaded (2012) y Díez (2012) contribuyen al estudio de las Tic promoviendo la necesidad de alfabetización mediática, suscitando la formación en y por medio de las Tecnologías, ampliando la perspectiva que los docentes tienen sobre su uso en el aula.

Según (Estrada, 2015) la calidad educativa es un tema recurrente en los ámbitos nacional e internacional, ya que está ligada, entre otras cosas, con la formación que se requiere que tenga el estudiante como futuro ciudadano para poder desempeñarse en forma adecuada en la sociedad actual, basada en la información. Dado este hecho, se ha considerado que las TIC son un elemento fundamental que puede potenciar la transformación de la calidad educativa, al integrar dichas herramientas en sus procesos pedagógicos de enseñanza-aprendizaje en pro de una educación contemporánea, alineada con los retos del tiempo moderno y que demandan los ciudadanos del siglo XXI. Basados en estos criterios es importante que la gestión tecnológica desarrollada en las entidades educativas se realice de forma correcta, considerando normas, estándares y buenas prácticas vigentes en la actualidad. Según (Hernandez, Gómez, \& Montenegro, 2014) las instituciones educativas son autónomas en la definición de sus planes de gestión de uso de TIC, pero tienen directrices generales que definen un marco general de gestión escolar. La autonomía de su funcionamiento no debería significar que no existan lineamientos ni métricas de evaluación para valorar los avances; al contrario, la planeación estratégica de TIC debe estar inmersa en el proyecto educativo institucional, brindando soporte en cada uno de los procesos claves para la Institución.

Desde hace algunas décadas, en muchos países se vienen implementando estrategias pedagógicas que permiten que, desde el inicio de la edad escolar, se gestione el conocimiento para así desarrollar habilidades sociales que permitan un aprendizaje basado en la retroalimentación con el entorno (Novoa-Ruiz, 2013).

(Cantor, 2014) menciona que al introducir la noción de calidad en la educación también se están involucrando aspectos coetáneos a los sistemas educativos, propios del mundo empresarial, tales como el control de calidad, mejoramiento de calidad, aseguramiento de la calidad. Con todas estas denominaciones tecnocráticas se está diciendo que la educación debe considerarse como una 
Rev. SINAPSIS, Edición № 12, Vol. 1, Diciembre 2018

ISSN 1390 - 9770

empresa, que produce mercancías, y esas mercancías deben estar sujetas a procesos de control que apunten a generar mejores resultados, que pueden ser cuantificados y estandarizados.

Para (Canto, Alí, Jaime, \& Uzcátegui, 2014), en la actualidad, las tecnologías permiten entregar herramientas que apoyan la gestión del conocimiento en las empresas, que apoyan la recolección, la transferencia, la seguridad y la administración sistemática de la información, junto con los sistemas diseñados para ayudar a hacer el mejor uso de ese conocimiento.

La capacitación y actualización docente en la formación de competencias digitales y en la renovación pedagógica para el uso y empleo de los medios tecnológicos. Salinas (1998) argumenta que la presencia de las tecnologías en la sociedad, transforman las instituciones educativas, configurándose un nuevo contexto, que conlleva una nueva necesidad de formación en los profesionales, para adaptarse a los nuevos cambios. Los nuevos espacios de enseñanza-aprendizaje exige la capacitación contínua de los docentes. (Lucia, Sara, Antonio, \& Dolores, 2015) “...la integración de las TIC en los proyectos educativos es entendida por los estados como una forma de lograr proyectos democráticos de inclusión y justicia"

Para (Alonso, 2007), las empresas que utilizan las TIC en la gestión de la información de sus procesos, se ha evidenciado que la principal deficiencia en sistemas de gestión integrados se encuentra en la incapacidad de gestionar los riesgos de seguridad de información. En la mayoría de las organizaciones se suelen presentar riesgos físicos y tecnológicos como elementos aislados, restándole importancia al impacto que causan en conjunto, ya que pueden influir en la Modelo integrado de gestión de riesgos de seguridad en los departamentos de TIC. productividad de las organizaciones.

En cuanto a las normas y/o estándares existentes para la gestión de TIC, resaltan los Objetivos de Control para la Información y la Tecnología Relacionada (COBIT), que según (González \& Molina, 2013), COBIT es un marco de referencia y un juego de herramientas de soporte que permiten a la gerencia cerrar la brecha con respecto a los requerimientos de control, temas técnicos, riesgos de negocio, y comunicar ese nivel de control a los interesados.

Según (Marulanda, López, \& López, 2009), el enfoque hacia procesos de COBIT subdivide las TIC en 34 procesos de acuerdo a las áreas de responsabilidad: planear, construir, ejecutar y 
monitorear; ofreciendo una visión de punta a punta de las TIC. Los conceptos de arquitectura empresarial ayudan a identificar aquellos recursos esenciales para el éxito de los procesos, es decir, aplicaciones, información, infraestructura y personas. COBIT da soporte al gobierno de TIC, al brindar un marco de trabajo que garantiza que: TIC está alineada con el negocio, TIC capacitan el negocio y maximiza los beneficios, los recursos de TIC se usen de manera responsable y los riesgos de TIC se administren apropiadamente Los productos COBIT se han organizado en tres niveles diseñados para dar soporte a: administración y consejos ejecutivos; administración del negocio y de las TIC; profesionales en gobierno, aseguramiento, control y seguridad.

Para (Santana, Rosalía, \& Jimbo Santana, 2017), uno de los planteamientos tradicionales para definir el buen Gobierno Corporativo (GC) es conseguir alinear el gobierno de Tecnologías de la Información (TI) con el negocio; pero es necesario definir una robusta y clara estructura de Gobierno Corporativo del que Tecnologías de Información forme parte indivisible. Cada día en mayor medida las organizaciones dependen de TI para cubrir las necesidades del negocio y crecer y/o, al menos, perdurar en su actividad.

Para esta investigación se consideró a una entidad educativa fiscal, es decir, financiada por el Estado, se trata de una institución ubicada en el cantón Tosagua, la misma que se encuentra ubicada en la provincia de Manabí, República del Ecuador. Para la realización de la presente investigación se consideraron los siguientes objetivos:

- Evidenciar el nivel de implementación de procedimientos internos para el uso de las TIC.

- Mostrar el nivel de conocimientos que tienen los usuarios para operar las TIC.

- Exponer la frecuencia con la que se capacita al personal en el uso de las TIC.

- Conocer los niveles de resolución de problemas y otros aspectos de la gestión de TIC

\section{Materiales y métodos}

En el presente trabajo de investigación se ha realizado un estudio de campo, con la finalidad de determinar el estado actual de la gestión de las TIC en una institución de educación secundaria del Cantón Tosagua, ubicada en la provincia de Manabí, República del Ecuador. 


\section{Rev. SINAPSIS, Edición №12, Vol. 1, Diciembre 2018}

Para realizar la investigación se consideraron 25 usuarios, que en este caso son docentes de la institución que utilizan los diferentes recursos tecnológicos. La encuesta incluyó una serie de preguntas que exploran la perspectiva de los usuarios acerca de la gestión interna de las TIC que se realiza por parte del encargado de dicha labor, para aplicar las encuestas se realizaron visitas previa autorización de la principal autoridad de la organización respetando sus principios institucionales, horarios y normativa interna.

La investigación se realizó para recopilar datos acerca de:

- El nivel de implementación de procedimientos internos para el uso de las TIC.

- El nivel de conocimientos que tienen los usuarios para operar las TIC.

- La frecuencia con la que se capacita al personal en el uso de las TIC.

- Los niveles de resolución de problemas y otros aspectos de la gestión de TIC.

\section{Resultados}

Una vez aplicadas las encuestas a los usuarios de TIC de la institución en mención, se obtuvieron los siguientes resultados:

\section{a. Nivel de implementación de procedimientos para el uso de las TIC}

El nivel de implementación de los procedimientos para el uso de las TIC desde la perspectiva de los usuarios se muestra a continuación:

\begin{tabular}{|c|c|c|}
\hline Alternativa & Frecuencia & Porcentaje \\
\hline $\begin{array}{c}\text { Procedimientos totalmente } \\
\text { implementados }\end{array}$ & 10 & $40 \%$ \\
\hline $\begin{array}{c}\text { Procedimientos parcialmente } \\
\text { implementados }\end{array}$ & 10 & $40 \%$ \\
\hline $\begin{array}{c}\text { No existe procedimientos } \\
\text { implementados }\end{array}$ & 5 & $20 \%$ \\
\hline Total & 25 & $100 \%$ \\
\hline
\end{tabular}

Tabla 1. Nivel de implementación de procedimientos para el uso de las TIC Fuente: usuarios de institución educativa secundaria 
Analizando las respuestas a las alternativas establecidas en esta tabla, se puede evidenciar que en esta institución educativa si existen procedimientos implementados total y parcialmente de acuerdo a las respuestas mayoritarias de los encuestados, mientras que por otro lado existe desconocimiento de parte de los usuarios sobre la implementación de estos procedimientos. Es necesario recalcar que cuando se implementa dentro de cualquier institución la gestión tecnológica, se facilitan todos los procesos organizacionales que vinculan al público interno y externo. "La gestión de tecnología es una de las disciplinas que en las pasadas tres décadas más se ha ocupado de estudiar y desarrollar la innovación empresarial, y la innovación tecnológica en particular" (Cabrera, 2010)

\section{b. Nivel de conocimientos de los usuarios para operar las TIC}

El nivel de conocimientos que tienen los usuarios para operar las TIC se muestra a continuación:

\begin{tabular}{|c|c|c|}
\hline Alternativa & Frecuencia & Porcentaje \\
\hline $\begin{array}{c}\text { Alto (Cuenta con título } \\
\text { académico relacionado con } \\
\text { TIC) }\end{array}$ & 1 & $4 \%$ \\
\hline $\begin{array}{c}\text { Medio (Cuenta con } \\
\text { experiencia en el uso de TIC) }\end{array}$ & 4 & $16 \%$ \\
\hline $\begin{array}{c}\text { Bajo } \\
\text { (Conoce aspectos muy } \\
\text { elementales para el uso de } \\
\text { TIC) }\end{array}$ & 20 & $80 \%$ \\
\hline No conoce & 0 & $0 \%$ \\
\hline Total & 25 & $100 \%$ \\
\hline
\end{tabular}

Tabla 2. Nivel de conocimientos de los usuarios sobre el uso de las TIC Fuente: usuarios de institución educativa secundaria

Analizando las respuestas a las alternativas establecidas en esta tabla, la mayor parte de los usuarios tiene bajos conocimientos sobre el uso de las TIC, sin embargo, aún existe un menor rango que si cuentan con experiencia en su uso debido a sus actividades diarias. Atendiendo a esta realidad del escaso conocimiento en TIC por parte de los usuarios de esta institución educativa y considerando que las aplicaciones de estas tecnologías dentro de las instituciones están en completo auge para lograr su desarrollo institucional, es menester conocer el nivel de la gestión tecnológica y empoderar a los usuarios de la institución en el óptimo conocimiento y aplicación de las TIC. “...ya que las mismas ocasionan un aumento en el nivel de competitividad de las organizaciones a nivel mundial, influenciado principalmente por las nuevas tecnologías, la calidad en los productos y 
Rev. SINAPSIS, Edición № 12, Vol. 1, Diciembre 2018

ISSN $1390-9770$

servicios, y la satisfacción del cliente como fin último de las organizaciones [VII]". (Jaimes Fuentes, Ramirez Prada, Vargas , \& Carrillo Caicedo, 2011)

\section{c. Frecuencia con la que se capacita al personal en el uso de las TIC}

La frecuencia con la que se capacita a los usuarios para que utilicen correctamente las TIC se muestra a continuación:

\begin{tabular}{|c|c|c|}
\hline Alternativa & Frecuencia & Porcentaje \\
\hline $\begin{array}{c}\text { Periódicamente (Por lo menos } \\
\text { una vez al año) }\end{array}$ & 3 & $12 \%$ \\
\hline $\begin{array}{c}\text { Esporádicamente (Por lo } \\
\text { menos cada dos años) }\end{array}$ & 20 & $80 \%$ \\
\hline No ha recibido capacitaciones & 2 & $8 \%$ \\
\hline Total & 25 & $100 \%$ \\
\hline
\end{tabular}

Tabla 3. Frecuencia con la que se capacita al personal en el uso de las TIC Fuente: usuarios de institución educativa secundaria

Analizando los resultados en gran medida la capacitación del personal en esta institución se lo realiza de manera esporádica por lo menos cada dos años; si bien es cierto todas las acciones que se ejecutan dentro de las instituciones se las realizan en base al reconocimiento de las debilidades y al robustecimiento de las fortalezas, situaciones concretas que se plantean en la planificación estratégica y que requiere de gestiones pertinentes en miras de conseguir los objetivos planteados. Según (Hernandez, Gómez, \& Montenegro, 2014), La autonomía de su funcionamiento no debería significar que no existan lineamientos ni métricas de evaluación para valorar los avances; al contrario, la planeación estratégica de TIC debe estar inmersa en el proyecto educativo institucional, brindando soporte en cada uno de los procesos claves para la Institución.

\section{d. Niveles de resolución de problemas y otros aspectos de la gestión}

Los niveles de resolución de problemas se muestran a continuación:

En cuanto al tiempo que se toma el encargado de TIC para atender problemas de los usuarios de TIC en la institución: 
Rev. SINAPSIS, Edición № 12, Vol. 1, Diciembre 2018

\begin{tabular}{|c|c|c|}
\hline Alternativa & Frecuencia & Porcentaje \\
\hline $\begin{array}{c}\text { Los problemas se resuelven } \\
\text { en menos de 1 hora }\end{array}$ & 0 & $0 \%$ \\
\hline $\begin{array}{c}\text { Los problemas se resuelven } \\
\text { de 1 a 3 horas }\end{array}$ & 4 & $16 \%$ \\
\hline Más de 3 horas & 21 & $84 \%$ \\
\hline Total & 25 & $100 \%$ \\
\hline
\end{tabular}

Tabla 4. Tiempos de respuesta del encargado de TIC para atender problemas de los usuarios

Fuente: usuarios de institución educativa secundaria

Analizando los resultados de esta tabla la capacidad de resolución delos problemas relacionados a las TIC se puede interpretar que aún no se alcanza el grado de satisfacción en cuanto al tiempo de respuesta a la solución del problema identificado, lo ideal sería que cualquier lograr la operatividad de la gestión tecnológica en el menor tiempo posible. Para (Hernandez, Gómez, \& Montenegro, 2014) las instituciones educativas son autónomas en la definición de sus planes de gestión de uso de TIC, pero tienen directrices generales que definen un marco general de gestión escolar.

En cuanto a la periodicidad con la que se realiza el mantenimiento de los equipos de la institución desde la perspectiva de los usuarios:

\begin{tabular}{|c|c|c|}
\hline Alternativa & Frecuencia & Porcentaje \\
\hline $\begin{array}{c}\text { Periódicamente (por lo menos } \\
\text { una vez cada seis meses) }\end{array}$ & 4 & $16 \%$ \\
\hline $\begin{array}{c}\text { Esporádicamente (más de seis } \\
\text { meses) }\end{array}$ & 19 & $76 \%$ \\
\hline Nunca & 2 & $8 \%$ \\
\hline Total & 25 & $100 \%$ \\
\hline
\end{tabular}

Tabla 5. Periodicidad con la que se realiza el mantenimiento de los equipos Fuente: usuarios de institución educativa secundaria

Analizando los resultados de esta tabla se puede evidenciar que no se está cumpliendo con la periodicidad en el mantenimiento de los equipos tecnológicos dentro de esta institución, ya que el mismo se lo realiza en un tiempo mayor a seis meses lo cual debilita la buena gestión tecnológica puesto que para lograr el buen funcionamiento de los equipos y poder brindar un servicio de calidad a los usuarios, es muy necesario realizar la revisión y el mantenimiento en un tiempo no mayor a seis meses. 
Rev. SINAPSIS, Edición № 12, Vol. 1, Diciembre 2018

ISSN $1390-9770$

En lo que se refiere al tipo de normas y/o estándares que utiliza la institución para la gestión de TIC

\begin{tabular}{|c|c|c|}
\hline Alternativa & Frecuencia & Porcentaje \\
\hline COBIT & 0 & $0 \%$ \\
\hline ITIL & 0 & $0 \%$ \\
\hline Otra & 0 & $0 \%$ \\
\hline No emplea ninguna & 19 & $76 \%$ \\
\hline No conoce & 6 & $24 \%$ \\
\hline Total & 25 & $100 \%$ \\
\hline
\end{tabular}

Tabla 6. Normas, estándares y/o buenas prácticas de gestión que emplea la institución. Fuente: usuarios de institución educativa secundaria

Analizando los resultados de esta tabla, se puede evidenciar que en la institución objeto de estudio, no se ha implementado un modelo de gestión donde se incluyan normas, estándares y/o buenas prácticas de la gestión de TI. ). Según (González \& Molina, 2013), COBIT es un marco de referencia y un juego de herramientas de soporte que permiten a la gerencia cerrar la brecha con respecto a los requerimientos de control, temas técnicos, riesgos de negocio, y comunicar ese nivel de control a los interesados.

\section{Discusión}

Este estudio se desarrolló considerando 25 usuarios de TIC de una institución educativa secundaria del cantón Tosagua, provincia de Manabí, República del Ecuador, siendo estos usuarios docentes que utilizan TIC en sus actividades diarias. El estudio se basó en la percepción de estos usuarios de TIC, en lo referente al nivel de implementación de procedimientos para que los usuarios puedan utilizar las TIC disponibles en la institución, el $40 \%$ afirmaron que están totalmente implementados, un $40 \%$ manifestaron que están parcialmente implementados, sin embargo, un $20 \%$ de los encuestados manifestaron que no existen procedimientos implementados, estos datos denotan que una gran mayoría de los usuarios de TIC tienen conocimientos sobre los procedimientos que deben emplear para poder utilizar las TIC correctamente.

Uno de los aspectos consultados en la encuesta, ha sido el nivel de conocimientos que tienen los usuarios para utilizar las TIC, en este caso sólo el $4 \%$ de los usuarios manifestaron tener conocimientos altos, es decir que cuentan con un título académico relacionado con las TIC, un 16\% 
Rev. SINAPSIS, Edición № 12, Vol. 1, Diciembre 2018

ISSN 1390 - 9770

manifestaron tener un conocimiento medio, es decir, que tiene experiencia en el uso de las TIC, sin embargo, el $80 \%$ de los usuarios encuestados manifestaron que tienen conocimientos bajos, es decir, que conocen aspectos muy básicos o elementales para el uso de las TIC, estos datos muestran que los usuarios no están debidamente capacitados y que no existe una política de capacitación que se esté aplicando para que los usuarios de TIC puedan aprovechar estos recursos de manera correcta.

Otro de los aspectos consultados fue la frecuencia con la que se capacita a los usuarios de TIC para que puedan utilizarlas correctamente, en este aspecto un $12 \%$ de los encuestados manifestaron que se realizan capacitaciones por lo menos una vez al año, sin embargo, el 80\% de los encuestados afirmaron que las capacitaciones se realizan aproximadamente cada dos años, situación confirma lo que ya se evidenció anteriormente acerca de la ausencia de una política de capacitación para mejorar el nivel de conocimientos de los usuarios en el uso de las TIC.

En lo referente a los aspectos que están más ligados con la gestión de TIC propiamente dicha, el $16 \%$ de los usuarios manifestaron que los problemas o incidentes ligados con las TIC se resuelven en un tiempo de entre 1 y 3 horas, sin embargo, el 84\% de los usuarios manifestaron que los problemas toman más de 3 horas en resolverse, situación que muestra altos tiempos de respuesta para la resolución de problemas por parte de los encargados de la gestión y, por lo tanto la incomodidad de los usuarios que necesitan de las TIC para desarrollar sus actividades.

Dentro de la gestión de las TIC, otro aspecto consultado fue la periodicidad con la que se realizan mantenimientos a los equipos, donde un $16 \%$ manifestaron que se realizan de manera periódica, es decir, por lo menos una vez cada seis meses, sin embargo, un 76\% de los encuestados manifestaron que dichos mantenimientos se realizan de manera esporádica, es decir, se realizan con demoras de más de seis meses, esta situación no asegura un funcionamiento apropiado de los equipos, y evidencia una clara ausencia de políticas y/o procedimientos que normen correctamente este aspecto de la gestión de TIC.

En este estudio también se consulto acerca de las normas y/o estándares que utiliza la institución para la gestión de TIC, en este aspecto el 76\% de los usuarios aseguraron que no se utiliza ninguna norma, y un 24\% manifestaron que no conocen del tema, es decir, que desde la perspectiva de los usuarios la institución no emplea ninguna norma, situación que demuestra la necesidad de 
Rev. SINAPSIS, Edición № 12, Vol. 1, Diciembre 2018

implementar mecanismos claros para la gestión de TIC, y por otra parte plantean la interrogante del por qué no se aplican las normas y/o estándares para este tipo de actividades.

\section{Conclusiones}

Al terminar la presente investigación de campo en la institución analizada sobre la gestión interna de TIC desde la percepción de los usuarios, se concluye:

- Para que las TIC puedan ser aprovechadas correctamente es necesario elevar el nivel de conocimientos de los usuarios en cuanto a su correcta utilización, esto se puede lograr empezando por planificar mejor las capacitaciones asegurando el perfeccionamiento progresivo de las habilidades de los usuarios de TIC.

- Dentro de la gestión de TIC los tiempos de respuesta para la resolución de problemas son altos, lo que puede derivar en molestias de los usuarios por cuanto no pueden continuar con sus actividades normales cuando necesitan de las TIC como apoyo fundamental para realizarlas.

- La periodicidad con la que se realiza el mantenimiento de los equipos no es la adecuada, esto se deriva de la ausencia de lineamientos claros para esta actividad.

- Se evidencia la ausencia de normas y/o estándares para la gestión de las TIC, lo cual es preocupante, ya que en la actualidad existen muchas entidades que se dedican a la generación de este tipo de documentos para hacer más eficiente dicha gestión.

\section{Referencias}

Acevedo, H. (2010). ITIL: ¿qué es y para qué sirve? (parte 1).

Agudelo, E., Lina, M., Niebles, A., \& Gallón, L. (2005). La gestión tecnológica como herramienta de planeación.

Alonso, R. C. (2007). Técnologías de la Información y la Comunicación. España: S.L.

Aronica, S., \& Peretti, M. (2010). Gestión tecnológica en las organizaciones: ¿quiénes deben asumir esta función?

Ávalos, E. (2003). Aproximación a la gerencia de la tecnología en la empresa. Estrategias, planificación y gestión de ciencia y tecnología. Editorial Nueva Sociedad. 
Rev. SINAPSIS, Edición № 12, Vol. 1, Diciembre 2018

ISSN 1390 - 9770

Cabrera, E. A. (2010). Gestión Tecnológica en Empresas Innovadoras Mexicanas. RAI - Revista de Administración e Inovación, 22.

Canto, E. D., Alí, G., Jaime, M., \& Uzcátegui, D. (2014). Gestión del conocimiento y uso de TIC’S en la empresa privada y administración pública. $R A G C, 17$.

Cantor, R. V. (2014). La calidad educativa una noción neoliberal, propia del darwinismo pedagógico. Integra Educativa, 13.

Estrada, J. (2015). Sistematización de un modelo de gestión para la incorporación de TICTecnologías de información y comunicaciones- en procesos educativos en municipios de Colombia.

González, L., \& Molina, C. (2013). Diseño del Modelo de Gestión de TIC's para los Infocentros de las Comunidades Indígenas.

Hernandez, U., Gómez, A., \& Montenegro, Y. (2014). Modelo de referencia para la planeación estratégica de TIC focalizada en las fases de verificación y realimentación en instituciones educativas de nivel básica y media.

Jaimes Fuentes, M. L., Ramirez Prada, D. C., Vargas , A. M., \& Carrillo Caicedo, G. (2011). Gestión Tecnológica: Conceptos y Casos de Aplicación. GTI, 12.

Lucia, N., Sara, C., Antonio, A. J., \& Dolores, M. M. (2015). Implicaciones, Uso y Resultados de las TIC en Educación Primaria. EDUTEC, 17.

María, D., \& Pere, M. (2013). Práctica Docente en Aulas 2.0 de Centros de Centros de Educación Primaria y Secundaria de España. Medios y Educación, 14.

Marulanda, C., López, M., \& López, C. (2009). Modelo de Desarrollo para Gobierno TI. Scientia et Technica Año XV, 6.

Navarro, K., Romero, E., Bauza, R., \& Granadillo, V. (2006). Estudio sobre la gestión tecnológica y del conocimiento en una organización creadora de conocimiento. 
Rev. SINAPSIS, Edición №12, Vol. 1, Diciembre 2018

Odremán, J. (2014). Gestión Tecnológica: Estrategias de Innovación y Transferencia de Tecnología en la Industria.

Santana, M. d., Rosalía, P., \& Jimbo Santana, A. E. (2017). Análisis de los Modelos de Capacidad para el proceso de gobierno de TI. Publicando, 8.

Thamhain, H. J. (2005). Management of technology. New Jersey. 